\title{
Filigrane
}

Écoutes psychothérapiques

\section{" Je ne vois de vous que ce que j’ai sous les yeux "}

\section{Marie Desrosiers}

Volume 18, numéro 2, automne 2009

Le corps. Sur le divan. Dans le fauteuil II

URI : https://id.erudit.org/iderudit/039285ar

DOI : https://doi.org/10.7202/039285ar

Aller au sommaire du numéro

\section{Éditeur(s)}

Revue Santé mentale au Québec

\section{ISSN}

1192-1412 (imprimé)

1911-4656 (numérique)

Découvrir la revue

\section{Citer cet article}

Desrosiers, M. (2009). « Je ne vois de vous que ce que j'ai sous les yeux ».

Filigrane, 18(2), 5-15. https://doi.org/10.7202/039285ar

\section{Résumé de l'article}

Ce texte présente un fragment clinique qui illustre un travail de passage permettant la fabrication d'une substance psychique utilisable pour l'analyse. Se situant dans une zone intermédiaire entre la mise en acte corporelle, la construction d'images et la représentation de mots, l'auteure tente de rendre compte d'un effet du symptôme d'une patiente sur ses propres constructions psychiques dans le cours de son travail avec celle-ci. L'auteure rend compte d'un travail d'élaboration qui vise à utiliser ses propres points d'ancrage dans la parole afin de limiter un effet d'attraction hors du champ de la représentation de mots auquel thérapeute et patiente se situent à l'approche d'un noyau traumatique. 


\title{
"Je ne vois de vous que ce que j'ai sous les yeux»
}

\author{
marie desrosiers
}

Ce texte présente un fragment clinique qui illustre un travail de passage permettant la fabrication d'une substance psychique utilisable pour l'analyse. Se situant dans une zone intermédiaire entre la mise en acte corporelle, la construction d'images et la représentation de mots, l'auteure tente de rendre compte d'un effet du symptôme d'une patiente sur ses propres constructions psychiques dans le cours de son travail avec celle-ci. L'auteure rend compte d'un travail d'élaboration qui vise à utiliser ses propres points d'ancrage dans la parole afin de limiter un effet d'attraction hors du champ de la représentation de mots auquel thérapeute et patiente se situent à l'approche d'un noyau traumatique.

\begin{abstract}
This paper presents a clinical fragment illustrating the transition towards the creation of psychic material suitable for analysis. Within an intermediary zone between corporal acting, image construction and word representation, the author attempts to report the effects of a patient's symptoms on her own psychic creations as the analytic work unfolds, the latter aiming to anchor the elaborative process in the use of words in order to ward off opposing tendencies taking both analyst and patient away from representation as they approach a traumatic nucleus.
\end{abstract}

À Patrick Cady

\section{La sculpture absente}

U ne sculpture absente de mon bureau s'est mise à occuper ma pensée, fugitivement en séances, puis obstinément en-dehors de celles-ci. Je me rappelai avec une sorte de plaisir empreint de nostalgie qu'elle était à moi. Les papiers attestant de mon droit à sa propriété étaient abrités quelque part, seul signe concret de sa présence réelle dans ma vie. Je m'en ennuyais comme d'un petit morceau d'environnement ayant peuplé mon enfance. À l'une de ses apparitions dans mon bureau, je m'assombris en pensant qu'elle était liée à la mort éventuelle de mon père qui la conservait, bien qu'il me l'ait déjà léguée. Au-delà de notre appréciation partagée de cette œuvre, elle se présentait aussi pour mon père en tant que souvenir intime d'une relation avec l'artiste qui avait martelé la pierre grise qui reposait aujourd'hui sur un socle de bois. J'ai imaginé que cette sculpture était l'une des pièces de réalité qui rappelait à sa mémoire le souvenir vivant d'un triangle amoureux avec deux sculpteurs, un lieu où lui-même avait trouvé la foi, l'inspiration, dans le lien à ces autres qui peuplaient l'un de ses mondes. Tout cela m'évoquait les liens qui unissent d'une étrange façon ceux qui sont liés par la mise en commun de leur créativité artistique, dans une expérience charnelle et spirituelle dont tous les autres semblent devoir s'exclure. Peut-être 
mon père avait-il investi ces artistes plus que ses enfants faits de chair, sans se le dire à lui-même, mais le démontrant dans la générosité de ses gestes, dans la passion de ses paroles et dans l'immense respect à leur égard.

Elle m'apparaissait, cette sculpture, comme issue d'un ailleurs dont j'avais été exclue. Il me paraissait curieux, avec cette patiente, d'oser ainsi rêver en catimini une sculpture qui me plaçait à nouveau dans ce désir paradoxal: vouloir cette oeuvre était-il associé à un désir autre qu'il est possible d'éprouver face à un père ? La sculpture venait d'abord représenter la suspension de mon désir, ce désir ne pouvait se faire que de loin, je ne pouvais que m'approprier cette oeuvre en pensée, sans sa présence matérielle sous ma main ni sous mon regard. Dans cette espèce d'hallucination en séance, il s'agissait d'une expérience curieuse et j'éprouvais à un point tel la présence et la solidité de la sculpture qu'elle me donnait à penser qu'une troisième dimension se matérialisait en moi, pour cette patiente-là. Elle s'érigeait là, dans le coin de mon bureau, droite, verticale et solide. Je n'aurais eu qu'à fermer les yeux pour l'imaginer là, à nouveau.

Au fond, ce n'est qu'après l'épisode de la sculpture que j'ai cru saisir, au cœur de ce parcours avec cette femme, ce qu'impliquaient réellement les propos de Fédida (2007) quand il réclame de l'analyste de se laisser transformer par le symptôme. À n'en pas douter, cela signifiait se laisser «vraiment» modifier, cela se posant davantage comme une condition qu'un bénéfice secondaire au travail d'analyse. Avec cette patiente qui était liée à l'apparition de la sculpture dans ma pensée, l'action d'un symptôme sur mes processus de pensée et sur ses contenus avait jusqu'ici transité par l'omniprésence de l'élément liquide. Le liquide est ce qui qualifiait autant la «tonalité» de l'univers psychique de cette analyse (affects, représentations, mises en acte corporelles) que ce que je me figurais d'un «tonus » traduisant une forme particulière de consistance (débits, formes de stagnation, écoulement). Quelque chose, maintes fois renommé, ne pouvait contenir une substance et celle-ci recevait aussi plusieurs noms. Les manifestations hémorragiques qui s'étaient emparées du corps de la patiente n'en étaient que l'un des signes.

Diverses images étaient venues offrir des variantes à un scénario témoignant de la disparition d'un contenant psychique et de l'écoulement de son contenu mais aussi, l'amorce de notre construction offrait ses propres repères. Une mère s'écoulait par des trous dans le cercueil; une fille aspergeait de son urine sa maman, lui donnant le cancer dont cette dernière n'allait jamais se remettre; une femme mettait en acte des scénarios de suicide, ne parvenant plus à succomber à l'attraction absolue d'être aspirée par un trou dans la terre; une mère glissant sur une flaque se répandait sur le sol, étalant son manque de consistance; une mourante était privée d'eau à ses dernières heures devant sa fille hébétée qui venait de laisser tomber la bouteille contenant le liquide salvateur. Ça semblait couler de partout. Par ailleurs, le symptôme avait aussi agi d'une façon particulière qui inversait constamment l'ordre des choses, on risquait toujours de se demander, dans une scène ou l'autre, s'il s'agissait de la mère ou de la fille ? de la mort ou du vivant? 
qui transmettait quoi et à qui ? Les liquides semblaient couler en tous sens, défiant la logique matérielle et une transmission paradoxale avait été figurée au fil du temps par la mise en image de transmissions sanguines, d'insertion de solutés où l'on ne sait plus vers où «ça» coule. Mettre en vie ou mettre à mort se situait toujours dans le même voisinage, dans une logique de l'eau trouble et de la contamination par les liquides, par ce qui s'écoule d'une à l'autre...

Le temps semblait venu pour que moi-même je m'arrache à un effet d'hémorragie psychique que cette patiente m'avait fait éprouver, longtemps à mon insu. Et cet effet hémorragique, il venait tout juste de se révéler à moi dans la période de l'avènement de la sculpture. Je devins tout particulièrement préoccupée par ce défi : dans cette traversée, comment allions-nous passer d'un état à un autre et que signifiait cette façon de le dire, «passer de l'état liquide à l'état solide », expression qui me semblait à moi-même énigmatique? Après l'épisode en question, la patiente offrit cette métaphore qui figurait peut-être un lieu où nous venions d'accéder. Elle s'attarda sur la constitution d'une aquarelle imaginée, se figurant les frontières fluides mais suffisamment distinctes que forment les couleurs qui empiètent les unes sur les autres, en laissant voir, par effet de transparence, des degrés de dilution. La sculpture, comme symbole de verticalité et de solidité, avaitelle pu contribuer à lui permettre à elle d'imaginer les dégradés témoignant d'une nouvelle force d'inscription?

\section{Mi-solide, mi-lisible}

Le passage du liquide vers le solide, ça ne me semble pas être le passage de la mère au père, ni le passage vers un statut particulier ou une fonction spécifique de la parole. Mais, d'une certaine façon, c'est aussi l'un et l'autre: un meilleur ancrage dans la fonction paternelle et une nouvelle possibilité d'écoute où la parole se présente davantage avec ses lieux de fuite qu'avec ses points de failles. Plus qu'une simple façon de nommer un climat, le fait d'avoir baigné pendant des mois dans un univers liquide n'était pas étranger à l'émergence subite de mes rêveries en séance. C'est ainsi que dans cette traversée aquatique, je me suis mise à sentir tout le poids psychique de la sculpture, comme le leste qu'on peut imaginer de la cale d'un navire et qui lui permet de ne pas tanguer. Nous flottions depuis déjà un bon moment, avec comme horizon, ce qui s'offrait comme des restes d'épaves dispersés après un désastre marin. Il aurait été difficile de déterminer si l'éparpillement des morceaux de scènes occupant la situation analytique traduisait uniquement un vécu traumatique et son effet de clivage sur la patiente. On pouvait aussi envisager que cet éclatement de pensée reflétait la fragmentation opérée par une écoute qui n'avait pas eu comme moteur le désir d'une reconstitution. Non, le processus de construction dans cette analyse avait suivi un autre cours, comme si l'apprentissage de la langue du symptôme avait requis toutes les énergies pour d'abord fabriquer une substance à l'analyse.

Peut-être l'histoire de cette sculpture, qui se tenait debout dans ma pensée, a-t-elle d'abord permis l'accrochage vers un ailleurs vertical. Elle venait s'offrir à 
moi comme point d'attache aux affects restés en flottement avec cette patiente qui, pendant des mois, m'avait fait visiter des pierres tombales sans noms, couchées à même le sol, ou s'érigeant dans des lieux d'une autre culture, dans une langue qui n'était pas la sienne, monuments publics d'une ville qu'elle avait déjà habitée. C'est avec cette femme que j'aurai eu à faire le trajet qui venait placer l'art non pas uniquement comme un objet de questionnement ou de curiosité, mais bien comme un élément nécessaire à la survie. Peut-être la sculpture aura-t-elle permis de garnir ma «trousse de sécurité » à ce moment de notre aventure, comme on traîne avec soi un petit morceau de civilisation en se disant que «ça pourra toujours servir...». Peut-être était-ce là, au tout début de son apparition, une des fonctions protectrices de cette sculpture, mettre au-dehors pour se soustraire à l'attraction pour le dedans? Après-coup, il me semble plutôt que la solidité espérée devait nous permettre de limiter momentanément un effet d'attraction, de manière à instaurer une force d'inscription. Lorsque la capacité imaginative des deux protagonistes de la situation analytique est mise face à un tel défi, comme celui de réanimer jusqu'à la matière inanimée, il ne reste qu'à espérer que la capacité à délirer et à devenir un peu fou, que cette étrange sagesse puisse enfin se mettre au service de la construction, pour le patient.

Peut-être faut-il concevoir, tout comme on imaginerait une mère qui peut advenir en tant que mère que parce qu'un nourrisson le provoque et l'exige, que le thérapeute tend lui aussi à advenir comme analyste, pour cette personne unique qui lui parle dans la situation tout à fait unique qui constitue l'espace analytique. Si le thérapeute accepte de se laisser former et déformer par le patient, et cela fait partie des héritages laissés par la clinique férenczienne, il n'a d'autre choix que de répondre à cette demande inconsciente d'analyse mutuelle que ce dernier lui livre. Mais répondre, il ne le fait que d'une certaine manière, c'est-à-dire en suspendant une réponse manifeste, afin de remettre en mouvement sa propre capacité d'accueil et d'adoption de l'autre. Il est face au devoir d'élaborer en lui-même cette demande d'analyse mutuelle et de s'offrir en tant que matériau malléable, ce que nous enseignent par ailleurs les paysages défrichés par les Winnicott, Fédida ou De M'Uzan, par exemple, dont l'ensemble des œuvres respectives témoigne de la nécessité, pour l'analyste, de faire éclater sa créativité. L'action du symptôme de cette patiente sur ma propre personne, action ne se limitant pas à ma psyché, avait ceci de particulier: me mettre au défi d'ouvrir les yeux, au sens propre et figuré. Là où le réel s'inscrivait de façon si marquante, il me fallut d'abord ouvrir mes yeux pour voir avant d'accepter de l'entendre, elle, c'est-à-dire d'apprendre à lire ce que moi-même j'avais mis sous ses yeux.

\section{«Je ne vois de vous...»}

«Je ne vois de vous que ce que j'ai sous les yeux», avait laissé échapper la patiente, juste un peu avant que notre attention se porte sur la toile qu'elle voyait du divan. Lorsqu'elle avait effectivement prononcé cette phrase, c'était plutôt à un moment où il lui semblait avoir vraiment aperçu mon visage, associé à un moment 
inaugural dans notre rencontre. Mais le jour où il fut question de la toile, elle s'était étendue en associant autour d'un thème se référant à ce qui est donné à voir, à ce qu'on a sous les yeux. Il aurait été possible d'entendre, par exemple, ce nouvel aperçu de mon corps, j'aurais pu utiliser des indices faisant penser que sa perception de ma présence physique devenait plus consciente, que ce qu'elle avait vu quelques jour auparavant pouvait lui rappeler autre chose, ou d'accentuer l'exclusion marquant le «je ne vois de vous que...». En bref, les mots actuels auraient pu être recueillis par divers lieux de mon écoute. Or, depuis cette séance de la toile, j'ai souvent repensé à l'imbrication des événements psychiques qui se sont mis en branle après la réponse que je m'étais alors entendue lui formuler. Ce jour-là, je n'avais pas emprunté la route toute tracée du corps. Il semble même qu'au lieu de symboliser quelque chose de son discours, j'avais plutôt posé un acte puisque, avant même d'ouvrir la bouche, j'avais d'abord levé les yeux en les posant, comme pour la première fois, sur le tableau qui nous faisait face à toutes les deux. «Que ce que j'ai sous les yeux...», cela résonna alors en moi et je soulignai que, à quelques reprises, elle avait introduit qu'il lui venait parfois à la pensée de voler quelque chose ici, parfois en évoquant prendre un tableau, sans jamais vraiment préciser lequel... Et c'est là, me semble-t-il, qu'elle commença à me parler de ce que nous avions, elle et moi, sous les yeux.

Elle se mit à commenter le tableau que j'avais accroché au mur qui lui faisait face, elle qui avait déjà fait une scène à propos d'un autre tableau que j'avais déplacé lors d'un déménagement du bureau, quelques années auparavant. Le tableau qui occupait la scène actuelle, sous nos yeux, me faisait occuper un nouveau rôle plus précis : celui de propriétaire. Elle venait de situer le peintre dont le nom se lisait facilement en tant que créateur me l'ayant légué. Enfin, quant à l'initiale du prénom sur la toile, sans aucun doute ni hésitation dans le cours de ses associations, il référait selon elle à l'initiale de mon propre légataire, une fille imaginée à qui je donnerais ce tableau. Une fille unique en était la destinataire puisqu'il était précisé sur un ton qui ne laissait place à rien d'autre «votre fille, votre vraie fille». C'est peut-être à ce moment- là que j'ai pu sentir concrètement combien mon hallucination de la sculpture n'avait pas été vaine puisqu'elle donnait un aspect encore plus réel à une inscription illisible qui avait déjà été mentionnée par cette patiente. De la sculpture à la toile, quelque chose dans notre dialecte semblait se transformer, comme si le détachement d'un usage habituel de la langue visant la communication ouvrait maintenant la voie à une nouvelle possibilité de fragmentation des matériaux psychiques à notre portée.

Toujours quelque peu distraite intérieurement par la sculpture, je me remémorai à cet instant l'importance des inscriptions absentes sur la pierre tombale de sa mère. Elle ne pouvait préciser le lieu exact où celle-ci reposait mais elle savait que le nom de sa mère n'y avait pas été gravé. Cette pierre était-elle couchée sur le sol ou se tenait-elle debout comme d'autres monuments dont il avait déjà été question? Indécidable: deux versions apparurent dans les semaines qui suivirent mais dans chacune des positions de cette pierre, aucune inscription, aucune 
marque d'identité, aucune trace de celle qui gisait peut-être sous terre. Le doute semblait encore possible, d'autant plus qu'elle n'avait pu assister à l'enterrement de sa mère. Elle n'avait pu que la voir dans son cercueil, cette mère si belle et si vivante, éternellement accessible par cette image la rendant disponible à tous fantasmes, livrant enfin son corps reposé et recomposé au regard inlassable de sa fille.

Devant la toile, celle-ci se mit au travail d'une façon insistante: on pouvait questionner l'attraction qu'exerçait l'initiale du prénom de la toile, comme si toute sa quête se déplaçait vers l'effort obstiné de déchiffrer, mais sans le dire ni rien me demander, l'unique initiale servant à indiquer un prénom sur cette toile. La force d'attraction se rattachant à cette lettre illisible pour elle semblait liée au fait que les initiales, c'est ce qu'elle partageait avec sa mère, elles avaient les mêmes, elles étaient en tous points pareilles, de ce point de vue là. Dans sa lecture de l'inscription indéchiffrable, une façon de dire insistait sur la position des lettres: l'initiale du prénom était incluse dans le nom de famille de son signataire, la petite lettre se lovait dans la majuscule. Par la magie de la langue, on aurait pu tout aussi bien inverser le mot qui avait été si souvent utilisé, lover, utilisé pour dire la position imaginée quand elle se reposait sur le divan ou même l'entendre à l'anglaise: lover comme aimer et se blottir. Quant à l'inversion qui venait dans cette écoute, la question de voler insistait dans ma pensée. Il avait souvent été question, sur le divan, d'être lovée dans le cercueil, aux côtés de la mère et il avait maintes fois été question de voler, soit des emblèmes de la mère ou des objets de l'environnement analytique.

Il s'ensuivit une nouvelle ébauche d'un scénario de vol qui déplaçait maitenant l'objet du vol imaginaire, une œuvre d'art, et qui faisait glisser cette prise d'objet vers un emprunt: prendre une des revues de la salle d'attente, avec la possibilité de me la remettre un de ces jours. Le contenu de la revue semblait secondaire et ce qui paraissait précieux à la patiente résidait encore ici dans ce qui était inscrit sur la petite étiquette collée en page couverture: mon nom et l'adresse du bureau y figuraient. C'est cette inscription qu'elle rêvait de transporter avec elle entre les séances, elle n'aurait eu qu'à ouvrir son sac à main pour se rassurer au sujet de l'existence de celle-ci. Ainsi prenait place une autre version de la preuve d'existence, comme des papiers d'une identité qu'on traîne sur soi. Ces preuves d'existence, la patiente avait depuis le début cherché à en créer, pour dire la preuve de vie ou de mort, dans sa tentative de laisser des traces de quelque chose qui risquait toujours de lui échapper. Il en allait ainsi de son sang, risquant toujours de laisser des traces sur le divan, dans la salle de bain, traces examinées attentivement pour dire leur degré de fluidité, leur couleur, l'apparition de substance plus ou moins solide, la naissance de caillots associés à la mort du fœus ou à l'espoir d'un meilleur endiguement du flot. Ce sang, il devenait une version parmi d'autres de l'univers que nous habitions depuis des mois, le corps étant parfois utilisé pour se dire. Le décentrement qu'avait provoqué ce temps de passage avait aussi permis que les hémorragies n'aient plus la même emprise sur moi, diminuant l'affolement 
qu'il m'arrivait d'éprouver. Il devenait possible de prendre acte du défi qui s'était alors posé à moi dans un corps à corps où se mêlaient le mort et le vivant, position qu'il m'avait fallu apprivoiser à petites doses. C'est peut-être là, au fond, que l'histoire de la sculpture avait joué un rôle ignoré jusqu'ici et qui me semble aujourd'hui avoir été fondamental en venant contrer la force d'attraction qui peut se vivre dans cette situation très particulière qui entremêle la mort à des forces érotiques très puissantes.

L'apparition de la sculpture et mon accrochage à quelque chose de «vraiment solide» aura peut-être favorisé une nouvelle rencontre avec ce que nous cherchions à nous approprier, indépendamment l'une de l'autre, en permettant un tissage commun qui dépendait de l'une et de l'autre, dans cette situation exclusive de l'analyse. Les semaines qui suivirent firent réapparaître jusqu'à quel point elle ne pouvait s'approprier quelque chose en lien avec son être et cela lui enlevait toute consistance. Cela la ramenait au fait de n'avoir pas été prénommée avant sa naissance et qu'il ne semblait pas y avoir de désir la précédant. Ces thèmes pouvaient alors être ébauchés sur de nouvelles bases, comme si le tableau avait figuré, le temps de l'élaboration, ce support absent pour que s'inscrive un nom.

\section{Construction paradoxale}

Avec la sculpture qui venait de faire son apparition dans ma tête, c'est comme si moi-même j'avais ouvert les yeux pour une première fois sur ces objets qui m'entouraient et qui peuplaient mon bureau et mon imaginaire. Ce n'est pas tant que je devenais forcée de voir, bien que je crois que les enfants qui ont été inondés par les productions artistiques d'un parent, je pense ici aux enfants de sculpteurs et de peintres, finissent parfois par s'infliger un aveuglement protecteur. Or, il m'a semblé intéressant de constater que jamais la patiente n'a évoqué la représentation de cette toile. Jamais non plus elle n'évoqua le nom de famille du peintre, peutêtre parce qu'il était le même que le mien. Il me vint que je n'avais jamais éprouvé si intensément que les termes «provocation du transfert par l'analyste» avaient leur juste place dans l'événement qui nous occupait elle et moi. Comment savoir si la force énigmatique pour la patiente prenait aussi sa source dans le fait qu'elle était tout simplement entrain d'ouvrir mes yeux, sur une autre scène, à propos de quelque chose qui m'avait si longtemps échappé. Le fait d'avoir exposé cette toile, ce qu'on pourrait juger comme un geste banal dans une logique matérielle de «l'aménagement intérieur», cela en vint à être traité par moi en tant que mise en acte que la patiente me révélait à mon sujet. C'est comme si elle me disait «vous avez mis sous mes yeux un support d'inscription mais aussi, ce que je vois de vous a force d'énigme». J'avais parfois l'idée que cette patiente me soufflait à l'oreille «ne vous défilez pas... nous jouons sur un terrain commun ».

La seule figuration de l'œuvre à laquelle elle s'était attachée résidait dans l'énigme de sa signature. Ses associations avaient été complètement absorbées à déchiffrer le nom d'un légataire, une «vraie fille». Avec ces patients en quête d'un désir au sujet de leur propre existence et dont l'esquisse d'une identité procède 
d'un choc traumatique des plus précoces, la question du «vrai» n'est jamais ni superflue ni banale et elle ne se limite pas à une question de vérité au sujet de ce que l'analyse pourrait leur faire découvrir quant à une histoire ancienne. Le vrai concerne bien souvent une demande d'authenticité qu'ils éprouvent au sein de la démarche actuelle avec l'autre, comme seul espoir d'y inscrire du vrai dans leur mémoire à venir.

Une seule fois, dans la suite de cet épisode, la patiente réagit massivement au contenu d'une toile nouvellement placée à l'entrée de mon bureau. Elle m'en parla comme on parle d'une personne, une entité ayant sa propre structure, sa personnalité. Elle était hors d'elle et je reçus comme un martèlement la longue interprétation qu'elle en fit, en essayant de ne pas m'extraire de son analyse puisque tout cela s'adressait à moi. Elle parla de ses lignes noires, de ses points de démarcation, de sa couleur et de sa dérangeante luminosité. J'en venais à éprouver l'impression d'avoir commis une obscénité en exposant ce tableau pourtant si «sobre». Elle le trouvait beau, trop beau. Essoufflée, elle avait pris un temps d'arrêt, peut-être est-ce là qu'avait surgi son sentiment d'indignation, quand elle s'était attaquée à ses dimensions. La toile, se disait-elle, n'aurait pas pu être camouflée, cela rendait son vol impraticable même en pensée.

Cette toile est de celles qu' on appelle «non figuratives» et cette abstraction aussi semblait l'avoir allumée. Tout cela, étrangement, me rassura. J'en venais à penser que la sexualité se trouve souvent dans des contenus ni génitaux, ni explicites, ni pornographiques et cela ouvrait la voie pour les mois à venir. Quant au non figuratif, il m'est personnellement difficile de concevoir une non représentation et le contenu de cette toile m'interpella tout particulièrement. Le tableau prétendument abstrait qui lui avait donné un choc pourrait être décrit comme une mosaïque. Il peut rappeler les vitraux qui laissent passer la lumière et mettent en vie des couleurs, celles-ci se trouvant bien encadrées par des traits noirs. S'il y a une manière de dire comment se présente la pensée de certaines personnes traumatisées, il me semble que la mosaïque est précisément une figuration qui vienne souvent à l'esprit. Cela rappelle Ferenczi qui, dans son journal clinique de 1932, évoque des constructions particulières et associées à une image incomplète, à la manière d'une mosaïque (Ferenczi, 1985, 70), chez certaines personnes traumatisées. Cela inaugure déjà ses élaborations sur certaines figures de l'effet du trauma telles l'atomisation et l'éclatement. Avec cette patiente, la survie d'un cadre, incluant la survie de l'autre, est probablement l'un des facteurs qui aura servi à tracer quelques traits noirs, donnant espoir que sa propre mosaïque pourrait se dessiner et être contenue, puisque nous approchions sans cesse d'un lieu où sa psyché semblait voler en éclats.

Ce lieu que nous abordions à répétition, en cherchant à fouler le terrain du traumatique, il rappelle aussi ce que Freud associa à un domaine inextricablement étranger et insuffisamment exploré (Freud, 1937, 256) dans ce texte où l'ombre de Ferenczi plane de façon à peine voilée. Freud s'y interroge entre autre à propos de patients qui présenteraient une certaine viscosité de leur libido. Il tente d'éclairer 
pourquoi ces personnes offrent parfois une fidélité d'investissement telle qu'ils ne parviennent pas à transporter vers un nouvel objet leurs investissements libidinaux restés accrochés ailleurs. Se référant à d'autres patients dont la libido, qui serait plus mobile, se montrerait plus favorable à de nouveaux investissements, Freud traduit ainsi sa pensée : "C'est la même différence que celle que peut bien ressentir le sculpteur selon qu'il travaille dans la pierre dure ou dans l'argile molle. Malheureusement les résultats analytiques dans ce second type se présentent souvent comme très fragiles; bientôt les nouveaux investissements sont eux aussi abandonnés, on a l'impression, non d'avoir travaillé dans l'argile, mais d'avoir écrit dans l'eau. » (Freud, 1937, 257) Encore la pierre, la glaise et l'eau... autant de matières par lesquelles les psychanalystes tentent depuis longtemps de faire entendre leurs conceptions, leurs désaccords, leurs constructions et leurs filiations.

\section{Sculpter dans la mélancolie}

Quelques saisons après l'événement de la sculpture, celle-ci me revint en mémoire et c'est alors que j'allais découvrir un sens nouveau à ce matériau psychique. Au moment où cela se produisit, la patiente se retrouvait dans un moment de régression comme cela n'était plus survenu depuis des mois. Son corps s'emparait alors de tremblements qui signalaient une lutte intense, sa respiration cadençait le drame qui semblait se jouer et sa voix nouée venait de formuler ce qu'elle semblait arracher péniblement au silence: «Je ne peux pas me lever, me mettre debout... » Elle avait mal dans son corps et elle tentait de dire l'angoisse de laisser derrière elle la morte, de tenir plus définitivement sa mère pour morte. Se mettre debout, élever son corps à la verticale, telles étaient les représentations d'un défi périlleux puisqu'il aurait, à son avis, signifié l'abandon du fantasme imaginé jusqu'ici à l'horizontale, dans un cercueil ou sur un divan. Le fantasme de se lover dans le corps de celle qui y était étendue, il révélait avec plus de netteté que jamais la position psychique qu'elle maintenait à son insu envers une mère à réanimer, à nourrir de sa substance.

Cela résonnait avec d'autres paroles déjà prononcées par la patiente au cours de notre périple, paroles qui nous avaient déjà laissées entrevoir différemment la fonction hémorragique, alors qu'elle s'était surprise elle-même en révélant «mais... je me saigne pour vous !». Or, le jour où elle faisait entrevoir l'impossible posture verticale, la patiente était confrontée au poids de son propre corps et à ce qu'elle y avait mis de solide avec frénésie, comme si se remplir et éprouver le plein étaient devenues les nécessités de la dernière année. C'est à partir de ce nouveau corps que la réécriture du fantasme des corps lovés semblait elle aussi avoir pris plus de consistance.

«C'est à sa mort et grâce à la mort», disait-elle, que la patiente avait enfin pu «faire un» avec la morte. Un corps courbé était venu en accueillir un autre. Une mère creuse permettait à sa fille de devenir son plein et depuis lors, celle-ci avait cherché à lui servir de substance complémentaire et nourrissante. Était-ce ce personnage logé dans son intérieur que la patiente avait si vaillamment nourri ces 
derniers temps? Cela rappelait le cannibalisme psychique dont certains cas de figure ont été finement présentés par Gabrielle Rubin (1997) alors que l'auteur semble travailler à l'ouverture de cryptes dont le corps réel s'est fait le contenant, la métaphore vivante.

Mais ce jour-là, alors que la représentation de ma sculpture prenait le pas sur sa fonction, me vint l'idée que je ne l'avais jamais laissée pivoter dans ma pensée, que je ne l'avais pas inclinée et que je ne l'avais donc pas vue sur un autre plan que celui de sa propre réalité. L'œuvre faite de pierre montrait un père qui porte à bout de bras son fils. Leurs corps se confondent mais la ligne ascendante de la sculpture donne force à ce soulèvement qui pourrait s'inverser : n'est-ce pas plutôt le fils qui vient tenir son père à bout de bras? Les formes sensuelles données à la pierre sont à peine figuratives et permettent toute inversion, comme dans le cas de certaines filiations paradoxales où l'on ne sait plus qui porte l'autre. Ce n'est qu'après la séance où l'angoisse transitait par «se mettre debout» que j'éprouvai une sorte d'étonnement face à la justesse de l'inconscient quand il s'emploie à produire ses propres images, nous laissant toujours libres d'y lire ou non quelque chose. Il m'apparaissait que j'avais eu sous les yeux ce qui s'était d'abord posé à moi comme un emblème de la filiation paradoxale et maintenant, je discernais encore plus nettement comment la sculpture proposait aussi une version verticale et solide du fantasme horizontal de la patiente. On aurait pu effectivement y voir deux corps lovés, se distinguant à peine l'un de l'autre, mais s'élevant selon une ligne puissante. Par ailleurs, ce n'est que plus tard encore que l'expression «tenir à bout de bras » m'est apparue comme une curieuse façon de dire qui fut ma façon pendant un temps pour tenter de faire passer le mouvement de cette sculpture au rang des mots. Or, tout comme c'est souvent le cas de certaines images de rêves que l'on tente de faire parler, l'expression que j'ai utilisée ne rend pas bien compte de l'appui du personnage tenu sur les jambes de celui qui le porte... à bout de bras ou à bout de jambes? «à bout de corps » me semblerait plus juste, maintenant.

Bien entendu, l'enjeu de tels parcours réside aussi dans un défi posé à la situation analytique qui risquerait d'induire en elle-même une dette sans fin. Pour le patient, l'espoir réside peut-être alors dans la possibilité que sa propre saignée, présentée comme nécessaire par lui-même, parvienne à atteindre son destinataire actuel dans la situation analytique. Et, éventuellement, que ce destinataire fasse entendre son renoncement à ce qui est conçu, sur le plan fantasmatique, comme une source devant le maintenir en vie. Les semaines à venir allaient permettre d'éclairer d'autres pans de ce qui avait rendu un deuil et une séparation impossibles jusqu'ici. Ainsi, un patient travail se poursuivit, portant d'abord sur le regard d'une mère à l'agonie, comme si cette scène venait confirmer le lieu de ce trauma au féminin se présentant comme une énigme béante dont il fallait retracer des contours. Un minutieux travail sur ce que l'œil avait saisi ouvrait ainsi la voie à ce qui avait été transmis à une enfant pour qui la conception des origines ne pouvait que passer par la conception de la fin. Ses théories sexuelles, celles de la mort, celles de la maladie ou de la vie s'étaient nourries aux mêmes scènes. Celles-ci 
portaient toutes les mêmes empreintes: celles de l'expérience d'être à la fois liquidée et pétrifiée, de même que le risque de se dissoudre et se liquéfier.

Comme un miroir, il avait aussi fallu inverser notre écoute, changer les personnages de l'énoncé qui s'était formulé par «je ne vois de vous que ce que j'ai sous les yeux ». Est-ce le regard de la mère posé sur sa fille qui avait un jour fait vomir la mère ? Est-ce le regard de la fille posé sur sa mère qui avait donné à la fille le goût de vomir, ce à quoi elle se refusait dans la réalité ? Les deux, peut-être, chacune des scènes servant sa propre fonction dans la mise en récit que tentait la patiente. Une situation traumatique prenait ici la figuration d'un miroir brisé et déjà, d'en assembler quelques morceaux, cela nous permettait de croire plus que jamais qu'une inscription, même lorsqu'elle ressemble au miroitement des reflets sur l'eau, peut devenir lisible, peut-être autant que lorsqu'elle nous semble gravée dans le roc. Sous la forme solide, le trauma pourrait ici nous faire penser davantage au diamant qu'à la perle, bien que cette dernière métaphore soit si souvent associée au noyau traumatique ${ }^{1}$. Mais si on le conçoit aussi comme il en serait de tenir à vue cette pierre précieuse, le travail à partir du trauma psychique révèle ses multiples facettes, nous fait toucher la démultiplication des images quand elles se sont pétrifiées. Mais tout cela donne à penser que c'est le diamant qui reste néanmoins le matériau naturel le plus dur, au point qu'il sert même à tailler le verre, et pourtant, c'est probablement le seul dont la transparence rappelle le mieux les reflets de l'eau...

marie desrosiers

583 , rue ladouceur

joliette (québec)

j6e 3 w4

desrosiersmarie@videotron.ca

\section{Note}

1. La perle qui est formée à partir du grain de sable que représenterait l'événement traumatique, une métaphore utilisée par S. Viderman, est réutilisé par C. Janin (1996) dans ses élaborations au sujet d'un noyau traumatique des processus psychiques.

\section{Bibliographie}

Fédida, P., 2007, Humain/Déshumain. L'oubli, l'effacement des traces, l'éradication subjective, la disparition, in Fédida, P. et. al., Humain/déshumain, Paris, Presses Universitaires de France, 11-124.

Ferenczi, S., 1985, Journal clinique, Paris, Payot.

Freud, S., 1937, L'analyse avec fin et l'analyse sans fin, in Résultats, idées, problèmes, 231-268.

Janin, C., 1996, Figures et destins du traumatisme, Paris, Presses universitaires de France.

Rubin, G., 1997, Cannibalisme psychique et obésité, Suisse, Delachaux et Niestlé. 